\title{
CHRONICALLY IMPLANTABLE, 121-CHANNEL, POLYMER MICROELECTRODE ARRAY WITH HERMETICALLY-SEALED WIRELESS INTERFACE

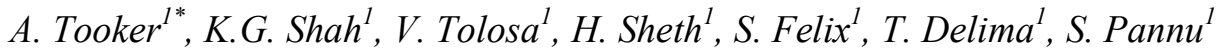 \\ ${ }^{1}$ Lawrence Livermore National Laboratory, Livermore, California, USA
}

\begin{abstract}
We present here the first demonstration of a 121-channel, chronically-implantable, wireless neural interface. This fullyimplantable device enables animals to be freely-behaving in their natural environment, unconstrained by external wires. This neural interface includes a polymer microelectrode array, capable of both stimulating and recording from neural tissue, integrated with a fully-biocompatible, hermetically-sealed, microelectronics package. The $121 \mathrm{recording} /$ stimulating electrodes demonstrate a high degree of reproducibility and repeatability.
\end{abstract}

\section{INTRODUCTION}

There is an ever growing need for reliable, biocompatible neural interfaces for chronic recording and stimulation of neural tissue. This capability is required for identifying and understanding the underlying mechanisms of the complex nervous system and developing neuroprosthetics to treat debilitating chronic neurological disorders such as deafness, blindness, spinal cord injury, depression, and several brain-related diseases. Most of the research used to investigate these conditions requires animals to be in their natural environment and not perturbed by the presence of personnel and the physical wires tethering them to measurement equipment. Thus, it is clear that a reliable penetrating neural interface with high electrode density and wireless telemetry is a critical technology component for scientific research and clinical applications. This work presents a fullyimplantable, wireless, neural interface for recording and stimulation of neural tissue. This neural interface consists of a polymer microelectrode array integrated with a microelectronics packaged that is hermetically sealed in a biocompatible package.

\section{Wireless Neural Interfaces}

Wireless neural interfaces have been developed by several groups [1-9] in recent years. There are four major elements that distinguish this work from previously published work.

1. The use of a polymer microelectrode array.

2. The microelectronics package is fully-implantable.

3. The microelectronics package is hermetically sealed.

4. There are 121 channels, all capable of stimulating and recording from neural tissue.

While each of these elements has been previously published [115], this is the first demonstration of the combination of all 4 of these elements. Similar demonstrations of these elements have been developed [8-9] primarily for use in retinal implants to restore site. These implants, however, have only 16-64 channels and are typically only capable of neural stimulation, not recording.

\section{Polymer Microelectrode Array}

There has been significant work done in the fabrication of both polymer [7-15] and silicon [1-6] microelectrode arrays for stimulation of and recording from neural tissue. These microelectrode arrays consist of discrete electrodes that are connected, via metal traces, to output leads and/or other signal processing circuitry. Neuronal stimulation and recording is conducted at these electrode sites.

Silicon microelectrode arrays [1-6] can be easily and efficiently fabricated, primarily due to the prevalence of silicon in the microelectronics industry and the resulting wealth of established fabrication techniques. Unfortunately, there are significant concerns regarding the suitability for long-term (i.e. chronic) in vivo studies, as silicon is not a biocompatible material [16]. Further, strain can be induced on the neural tissue due to the mismatch between the mechanical properties of silicon and neural tissue and the continuous micro-motion of the tissue. This can cause chronic injury and glial scarring at the implantation site.

Polymer microelectrode arrays [7-15] are an attractive alternative. The rapid development of MEMS fabrication techniques and the increasing presence of these polymers ensures that polymer-based microelectrode arrays can be fabricated with the same ease and efficiency as silicon microelectrode arrays. The mechanical properties of these polymer microelectrode arrays are more closely matched to that of neural tissue, thus imparting less strain and minimizing injury and glial scarring at the implantation site [17]. In addition, these polymers, specifically polyimide and parylene, are both biocompatible materials; thus, they are more suitable for chronic implantation than silicon [18].

Several wireless neural interfaces have been developed using silicon [1-6] and polymer [7-9] microelectrode arrays. The microelectrode array presented here is a polyimide microelectrode array.

\section{Fully-Implantable Microelectronics Package}

The microelectronics package presented here is fullyimplantable. Previous work (mostly retinal implants) has demonstrated the use of a fully-implantable microelectronics package [1,4,7-9]. Several groups, however, have used percutaneous or head-mounted microelectronics package [2-3,5-6]. There are two significant advantages for a fully-implantable package, especially for chronic in vivo studies. First, as the packages are fully-implantable they are less prone to breakage or damage. Second, fully-implantable packages are less prone to infection, as there are no open wound/surgical insertion sites.

\section{Hermetically-Sealed Microelectronics Package}

In addition to being fully-implantable, the microelectronics package is hermetically-sealed. Some previous work has demonstrated the use of a hermetically-sealed microelectronics package, although this has been limited to retinal implants [7-9]. The alternative to hermetic sealing is a polymer coating [1,4]. Polymer-coated microelectronics packages, however, are susceptible to sealing problems due to both potential nonuniformities in polymer coatings and adhesion issues. Hermetically-sealed microelectronics packages do not suffer from either coating non-uniformity or material adhesion problems; thus, they are more suitable for chronic, long-term in vivo studies.

\section{1-Channels for Neural Stimulation and Recording}

In general, more channels (i.e. electrodes) correlates to an increased number of neurons as potential targets for either stimulation or recording. Most wireless neural interfaces are limited to 15-16 channels [2-3,5-8]. Recently, several groups have demonstrated larger channel numbers, generally 60-100 channels $[1,4,9]$. We present here the first demonstration of a 121-channel wireless neural interface. 


\section{WIRELESS NEURAL INTERFACE - FABRICATION AND ASSEMBLY}

There are three main components to this wireless neural interface: 1) polymer microelectrode array, 2) hermetically-sealed microelectronics package, and 3) inductive coils for power and data transfer.

\section{Polymer Microelectrode Array}

Although the use of flexible polyimide minimizes the injury and glial scarring caused by the implantation of the microelectrode array, it does not eliminate it. The amount of injury and scarring can be further reduced by minimizing the size of the implanted microelectrode array. Unfortunately, typical efforts to minimize the size also limit the number of stimulation and recording sites (electrodes). In order to effectively decrease the size of the probe, while maintaining the electrode count, additional trace metal layers are required. Generally, the width of the microelectrode arrays is $20-30$ times the thickness. Thus, although the additional metal layers necessitate additional polyimide insulating layers, a significant decrease in microelectrode array width can be achieved with only a marginal increase in thickness. The resulting reduction in cross-sectional area will cause less injury and scarring while maintaining the same number of electrodes.

There are several examples of polyimide microelectrode arrays with one layer of trace metal [7-11]. In addition, some work has demonstrated the use of two layers of trace metal [12-15]. We present here a fabrication process for microelectrode arrays utilizing three layers of trace metal.

The general fabrication process for these 3-layer metal microelectrode arrays is summarized in Figure 1. The processing steps are summarized below:

1. Polyimide 1 Deposition $(5 \mathrm{~m})$

2. Deposition/Patterning of Trace Metal $1(\mathrm{Ti} / \mathrm{Au} / \mathrm{Ti})$

3. Polyimide 2 Deposition $(2 \mathrm{~m})$ and Inter-layer Via Etching $\left(\mathrm{O}_{2}\right.$ Plasma $)$

4. Deposition/Patterning of Trace Metal $2(\mathrm{Ti} / \mathrm{Au} / \mathrm{Ti})$

5. Polyimide 3 Deposition $(2 \mathrm{~m})$ and Inter-layer Via Etching $\left(\mathrm{O}_{2}\right.$ Plasma $)$

6. Deposition/Patterning of Trace Metal $3(\mathrm{Ti} / \mathrm{Au} / \mathrm{Ti})$

7. Deposition/Patterning of Electrode Metal (Ir)

8. Polyimide 4 Deposition $(5 \mathrm{~m})$ and Electrode Via Etching $\left(\mathrm{O}_{2}\right.$ Plasma)

9. Device Outline Etching $\left(\mathrm{O}_{2}\right.$ Plasma $)$

We have demonstrated this process using polyimide. The same fabrication process can be used with other polymers, such as parylene and silicones.

Photographs of the three-layer metal, polyimide microelectrode array are shown in Figure 2. The polymer microelectrode array consists of 5 distinct probes, all routed together and joined at a single connector. Each probe has 4 shanks; each shank has 6 recording/stimulating electrodes plus 1 reference electrode. This creates a single polyimide microelectrode array with 121 electrodes (all of the reference electrodes are shorted together). The flexibility of the individual probes ensures the final device can be easily assembled into a three-dimensional superstructure ( 4 x 5 array of shanks).

\section{Hermetically-Sealed Microelectronics Package and Inductive Coils}

The microelectronic components need to be hermetically sealed and separated from the neural tissue for two basic reasons. First, the electronics are made of non-biocompatible materials that may elicit a cytotoxic response from the neural tissue. Second, the electronics would corrode (and be shorted out) due to moisture and the harsh, biological environment of the body. Therefore, the microelectronics package consists of a biocompatible metal can attached to a ceramic substrate. The electronics are then sealed inside this can (Figure 3), effectively preventing physical interaction with the neural tissue.
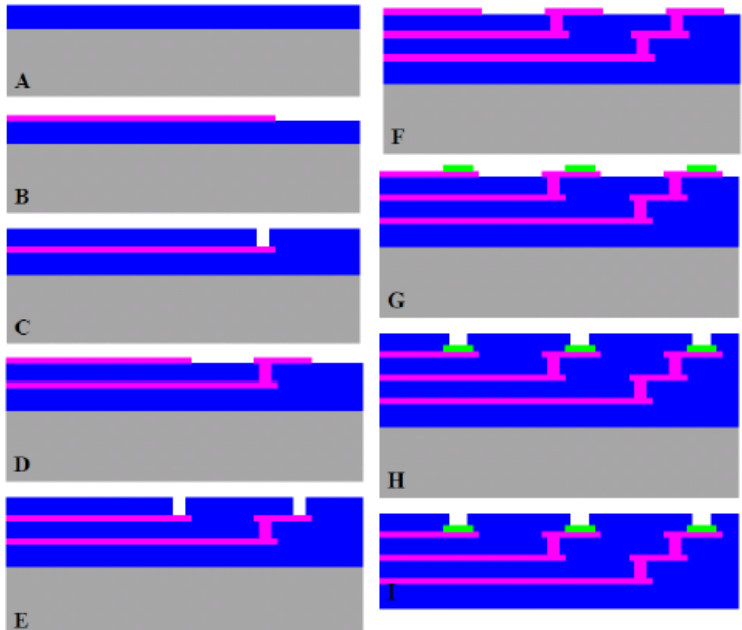

Silicon Polyimide

$\mathbf{T i} / \mathbf{A u} / \mathbf{T i}$

Ir

Figure 1: Cross-sectional view of the general fabrication process for three-layer trace metal, polyimide microelectrode arrays. (Images are not drawn to scale.)

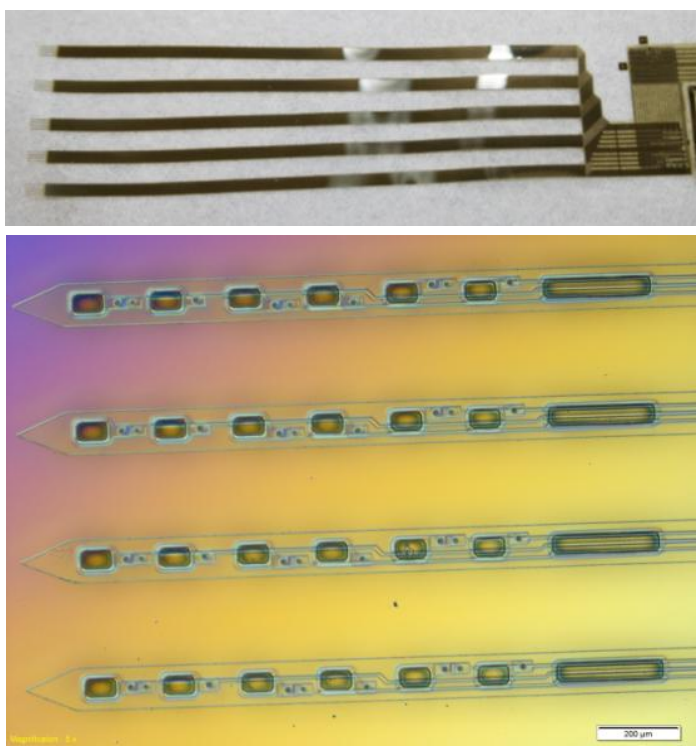

Figure 2: The entire polyimide microelectrode array, with 5 probes, is shown at the top. All of the probes are joined at a single connector (right). An enlargement of one of the probe tips (bottom) shows the 4 shanks, each with 6 electrodes for recording/stimulating and 1 reference electrode.

A variety of electronic components are used to convert the wireless data and power signals into the 121 individual electrical impulses that are delivered to the neural tissue. The electronics contained in this wireless neural interface are:

1. A power telemetry chip that converts the incoming power telemetry signal into the necessary voltages that operate the stimulator chip.

2. A stimulator chip that uses the incoming data signal 
and generates the independent electrical impulses. (This chip is scalable to 256 channels.)

3. Associated surface mount circuitry (both active and passive), such as an oscillator (for the chip clock), resistors, capacitors, diodes, and an inverter.

This wireless neural interface does not contain a battery, rather, the wireless data and power signals are transferred to it using inductively coupled coils. The inductive coils for wireless telemetry (power and data transfer) are fabricated using gold wire with polyimide coating for insulation.
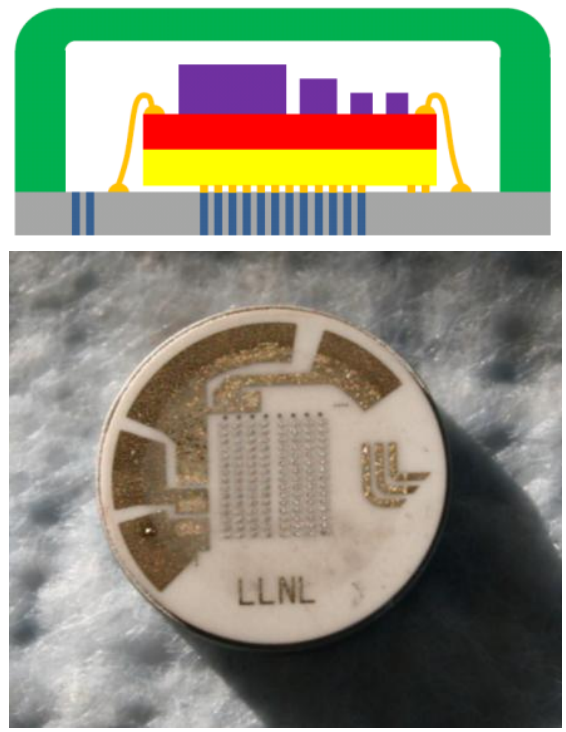

Figure 3: Cross-sectional schematic of the microelectronics package (top). The electronics are hermetically-sealed inside the metal can (green). The stimulator and power telemetry chips (yellow), silicon interconnect board (red), and surface mount circuitry (purple) are shown. The metal can (13 mm diameter, 3.8 $\mathrm{mm}$ tall) is brazed on a ceramic disc (gray) with high-density vias (blue) for connections between the electronics and the microelectrode array.

The electronic components are assembled using several microelectronics packaging/assembly methods in a manner that minimizes the total volume of the device. These components are assembled inside a hermetically-sealed package. The package consists of a ceramic substrate that contains an array of hermetic electrical feedthroughs which transmits electrical signals to and from the electronics and microelectrode array. The ceramic substrate is uniformly coated with a biocompatible metal stack. The metal is then lithographically-patterned on one side for the attachment of the electronics components and on the other side for the attachment of the polymer microelectrode array and the inductive coils. The stimulator chip is assembled directly on the ceramic substrate with flip-chip bonding. The remainder of the electronics components (power telemetry chip and other circuitry) are assembled onto a silicon interconnect board. High-aspect-ratio wire-bonds serve as interconnects between the power telemetry chip, the interconnect board, and the stimulator chip. The ceramic substrate is brazed to a biocompatible niobium ring. Once all of the electronics components are assembled onto the ceramic substrate, the microelectronics package is then hermetically-sealed by laser welding a niobium cap to the ring. Finally, the microelectrode array and inductive coils are connected to the ceramic substrate with gold rivet bonds [19] (Figure 4).

\section{NEURAL INTERFACE CHARACTERIZATION Electrochemical Testing}

The iridium activation was performed using biphasic potential pulsing in phosphate-buffered saline to form an activated iridium oxide film (AIROF). Individual iridium electrodes were characterized to determine charge storage capacity (CSC) and impedance both before and after activation. Cyclic voltammetry (CV) and electrochemical impedance measurements were made with a Princeton Applied Research (PAR) potentiostat using vendor-supplied software. All measurements were made in a three-electrode cell using a $\mathrm{Pt}$ counter electrode, an $\mathrm{Ag} / \mathrm{AgCl}$ reference electrode, and phosphate-buffered saline $(\mathrm{pH} 7.4)$ as the electrolyte. Potential cycling for the CVs was performed between $-600 \mathrm{mV}$ and $+800 \mathrm{mV}$ at a scan rate of $100 \mathrm{mV} / \mathrm{s}$.

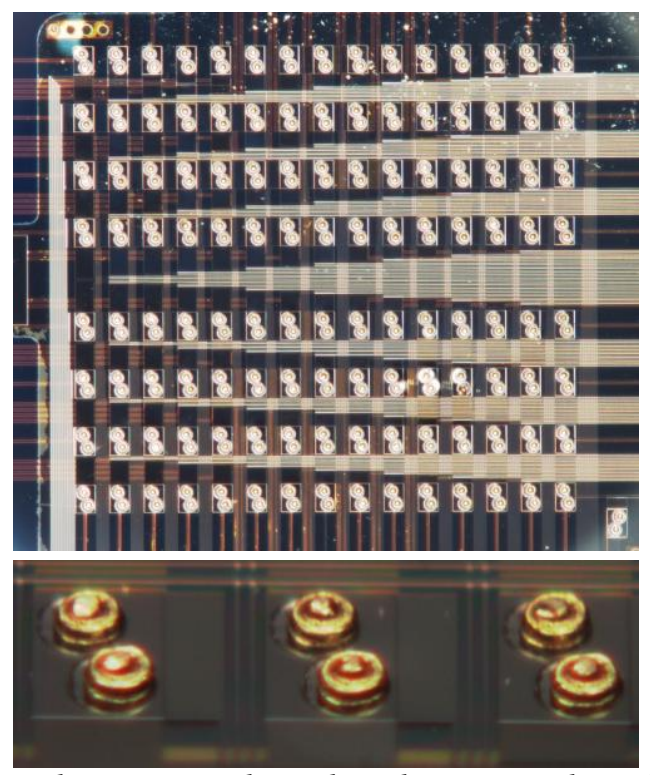

Figure 4: The top image shows the polymer microelectrode array with gold rivet bonds to the ceramic substrate. The bottom image shows an enlarged view of three rivet bond connections. Each electrode has two rivet bonds (for redundancy). Each pair of rivet bonds has a pitch of $305 \mu \mathrm{m}$. The rivet bonds are each $\sim 80 \mu \mathrm{m}$ in diameter.

Typical CV curves for 12 of the 121 electrodes are shown in Figure 5. The recording/stimulating electrodes are $40 \mathrm{~m}$ by 65 $\mathrm{m}$. As can be seen, the CVs for the different recording/stimulating electrodes overlap quite well and the characteristic iridium oxide peaks are clearly visible after activation. Post-activation, the average CSC for the recording/stimulating electrodes on a single device is 30.81 $\mathrm{mC} / \mathrm{cm}^{2}$, with a standard deviation of $0.70 \mathrm{mC} / \mathrm{cm}^{2}$. Postactivation, the average impedance for the recording/stimulating electrodes on a single device is $46.00 \mathrm{k} \Omega$, with a standard deviation of $4.52 \mathrm{k} \Omega$ (at a frequency of $948.7 \mathrm{kHz}$ ). Thus, for a single device, there is no significant difference in electrode characteristics.

\section{Biocompatibility Testing}

The selection and evaluation of materials and devices intended for use in humans requires a structured program of assessment to establish biocompatibility and safety. These wireless interfaces have passed the ISO-10993 array of biocompatibility testing for chronically implanted devices, in accordance with the FDA regulations. This includes assessing the 
biological reactivity, allergenic potential, sensitizing capacity, systemic toxic effects, immune response, and genetic mutations in animal models. The completely assembled interfaces (although with platinum electrodes), including microelectronics package, have successfully passed this biocompatibility testing. The neural interfaces with iridium electrodes have not been subjected to the complete array of testing, but have successfully passed basic cytotoxicity screening. We expect the neural interfaces with iridium electrodes to also successfully pass biocompatibility testing, due to the inherent similarities in material selection and fabrication process.

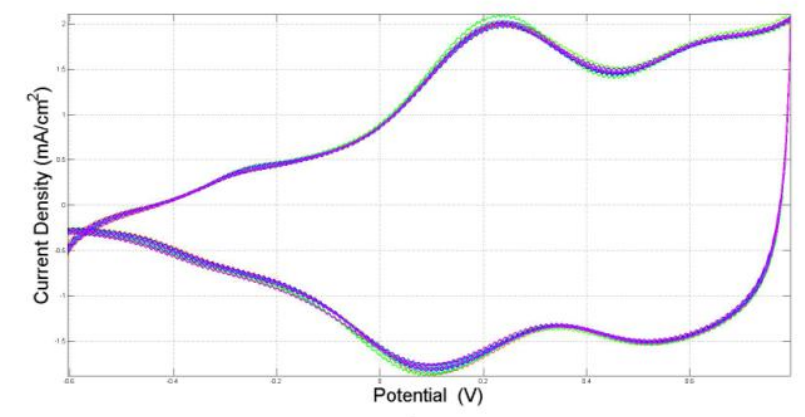

Figure 5: Typical CV curves of 12 representative recording/stimulating electrodes after electrochemical activation. The CVs overlap extremely well and the characteristic iridium peaks are clearly visible.

\section{CONCLUSION}

We have presented here the first demonstration of a 121channel, fully-implantable, wireless, neural interface for recording and stimulation of neural tissue. The neural interface consists of a polymer microelectrode array integrated with a hermeticallysealed, biocompatible, microelectronics package. The fully assembled neural interfaces are currently undergoing further functional and lifetime testing in preparation for in vivo studies.

\section{ACKNOWLEDGEMENTS}

We would like to thank Professor Wentai Liu's group at the University of California, Santa Cruz, for the microelectronics.

This work performed under the auspices of the U.S. Department of Energy by Lawrence Livermore National Laboratory under contract DE-AC52-07NA27344. LLNL-CONF544073

\section{REFERENCES}

[1] A. Sharma, L. Rieth, P. Tathireddy, R. Harrison, H. Oppermann, M. Klein, M. Topper, E. Jung, R. Normann, G. Clark, and F. Solzbacher, "Long term in vitro functional stability and recording longevity of fully integrated wireless neural interfaces based on the Utah Slant Electrode Array," J. Neural Eng., 8 (4), (2011).

[2] E. Greenwald, M. Mollazadeh, C. Hu, W. Tang, E. Culurciello, and N. Thakor, "A VLSI Neural Monitoring System With Ultra-Wideband Telemetry for Awake Behaving Subjects," IEEE Trans. Biomed. Cir. Sys., 5 (2), pp. 112 118, 2011.

[3] D. Fan, D. Rich, T. Holtzman, P. Ruther, J. Dalley, A. Lopez, M. Rossi, J. Barter, D. Salas-Meza, S. Herwik, T. Holzhammer, J. Morizio, and H. Yin, "A Wireless MultiChannel Recording System for Freely Behaving Mice and Rats," PLoS ONE, 6 (7), e22033 (2011).
[4] C. Chestek, V. Gilja, P. Nuyujukian, J. Foster, J. Fan, M. Kaufman, M. Churchland, Z. Rivera-Alvidrez, J. Cunningham, S. Ryu, and K. Shenoy, "Long-term stability of neural prosthetic control signals from silicon cortical arrays in rhesus macaque motor cortex," J. Neural Eng., 8 (4), (2011).

[5] A. Bonfanti, G. Zambra, G. Baranauskas, G. Angotzi, E. Maggiolini, M. Semprini, A. Vato, L. Fadiga, A. Spinelli, and A. Lacaita, "A wireless microsystem with digital data compression for neural spike recording," Microelec. Eng., 88, pp. $1672-1675$ (2011).

[6] M. Azin, D. Guggenmos, S. Barbay, R. Nudo, and P. Mohseni, "A Miniaturized System for Spike-Triggered Intracortical Microstimulation in an Ambulatory Rat," IEEE. Trans. Biomed. Eng., 58 (9), pp. 2589 - 2597, (2011).

[7] A. Sodogar, G. Perlin, Y. Yao, K. Najafi, and K. Wise, "An Implantable 64-Channel Wireless Microsystem for SingleUnit Neural Recording," IEEE J. Solid-State Cir., 44 (9), pp. $2591-2604$ (2009).

[8] S. Kelly, D. Shire, J. Chen, P. Doyle, M. Gingerich, W. Drohan, L. Theogarajan, S. Cogan, J. Wyatt, and J. Rizzo, "Realization of a 15-Channel, Hermetically-Encased Wireless Subretinal Prosthesis for the Blind," IEEE-EMBS Conf., pp. 200-203 (2009).

[9] http://www.artificialretina.energy.gov

[10] S. Lee, J. Jung, Y. Chae, J.-K. Suh, and J. Kang, "Fabrication and characterization of implantable flexible nerve cuff electrodes with Pt., Ir, and IrOx films deposited by RF sputtering," J. Micromech. Microeng., 20 (3), (2010).

[11] B. Rebehn, C. Bosman, R. Oostenveld, P. Fries, and T. Stieglitz, "A MEMS-based flexible multi-channel ECoGelectrode array," J. Neural Eng., 6 (3), (2009).

[12] A. Mercanzini, K. Cheung, D. Buhl, M. Boers, A. Maillard, P. Colin, J.-C. Bensadoun, A. Bertsch, and P. Renaud, "Demonstration of cortical recording using novel flexible polymer neural probes," Sens. Actuators A, 143, pp. 90-96 (2008).

[13] J. Seymour, N. Langhals, D. Anderson, and D. Kipke, "Novel multi-side, microelectrode arrays for implantable neural applications," Biomed. Microdevices, 13, pp. $441-452$ (2011).

[14] T. Doerge, S. Kammer, M. Hanauer, A. Sossalla, S. Steltenkamp, "Novel method for flexible double sided microelectrode fabrication process," Bioengineered and Bioinspired Systems IV Book Series: Proc. Of SPIE, 7365 (2009).

[15] T. Stieglitz, "Flexible biomedical microdevices with doublsided electrode arrangements for neural applications," Sens. Actuators A, 90 pp. 203-211 (2001).

[16] R. Biran, D. Martin, and P. Tresco, "Neuronal cell loss accompanies the brain tissue response to chronically implanted silicon microelectrode arrays," Exp. Neurology, 195, pp. 115-126 (2005).

[17] V. Polikov, P. Tresco, and W. Reichert, "Response of brain tissue to chronically implanted neural electrodes," J. Neurosci. Meth., 148, pp. 1-18 (2005).

[18] Y. Sun, S. Lacour, R. Brooks, R. Rushton, J. Fawcett, and R. Cameron, "Assessment of the biocompatibility of photosensitive polyimide for implantable medical device use," J. Biomed. Mat. Res. A, 90A (3), pp. 648-655 (2009).

[19] T. Stieglitz, H. Beutel, and J.-U. Meyer, "Microflex-A New Assembling Technique for Interconnects," J. Intelligent Mat. Sys. And Struc., 11 (6), pp. 417-25 (2000).

\section{CONTACT}

*A. Tooker, tel: +1-925-422-2326; tooker1@1lnl.gov 